\title{
Star Clusters in the Magellanic Type Irr Galaxy NGC 4449
}

\author{
Patrick Seitzer \\ University of Michigan, Dept. of Astronomy, 818 Dennison Bldg, Ann \\ Arbor, MI 48109, USA
}

Eva Grebel

Lick Observatory, Univ. of California, Santa Cruz, CA 95064, USA

\begin{abstract}
We compare the luminosity function of star clusters in the LMC and NGC 4449: a IBm/Sm type galaxy very similar in appearance and luminosity $\left(M_{B}=-18.5\right)$ to the LMC $\left(M_{B}=-18.4\right)$. The brightest of several hundred clusters in NGC 4449 have $M_{V}=-10$ (assuming a distance of $3.9 \mathrm{Mpc}$ ), comparable to the brightest clusters in the LMC.
\end{abstract}

How unique is the population of star clusters in the LMC? Do all Magellanic type galaxies have a similar system of star clusters? Or does it depend on environment, and any possible interactions the parent galaxy has endured? Although there have been many studies of clusters in members of the Local Group (NGC 6822, WLM, and others), most of these systems are considerably less luminous than the LMC. Indeed, in Harris' (1991) review of globular cluster systems in galaxies, there is no mention made of any clusters in galaxies similar to the LMC. Thus it is impossible to determine if the LMC cluster system is unique.

For comparions, we studied the clusters in NGC 4449, which is classified as $\mathrm{IBm} / \mathrm{Sm}$ in the Second Reference Catalog of Galaxies system, and is at a distance of $3.9 \mathrm{Mpc}$ (Melisse \& Israel 1994) with $M_{B}=-18.5$. Visually, it appears very similar to the LMC. This galaxy has been studied frequently because of a burst of recent star formation (Hunter \& Gallagher 1997; Hill et al. 1994; Bothun 1986). It is isolated on the sky: the nearest large galaxy is NGC 4258 which is over $3^{\circ}$ projected separation. But Hunter et al. (1998) show that gas in the system is highly disturbed in the outer parts, evidence perhaps for a recent interaction.

Analysis of WFPC2 archival images showed several hundred extended objects centered on the galaxy, which we interpret to be the star cluster population of NGC 4449. A total of 97 such objects brighter than $V=21$ were classified as star clusters based on their radial profiles. The sample will be incomplete at fainter magnitudes, in the center of the galaxy, and for those clusters with half light radii smaller than several WFC pixels $(2 \mathrm{pc}$ at $3.9 \mathrm{Mpc})$.

It is evident that NGC 4449 has a substantial number of rich star clusters, the brightest of which are comparable to the brightest in the LMC. The figure shows the observed luminosity function of the NGC 4449 clusters along with two estimates of the LF for the LMC clusters. Because the NGC 4449 sample is much more incomplete than the LMC samples, there will be a scaling factor in $\log (\mathrm{N})$ which still needs to be determined. 


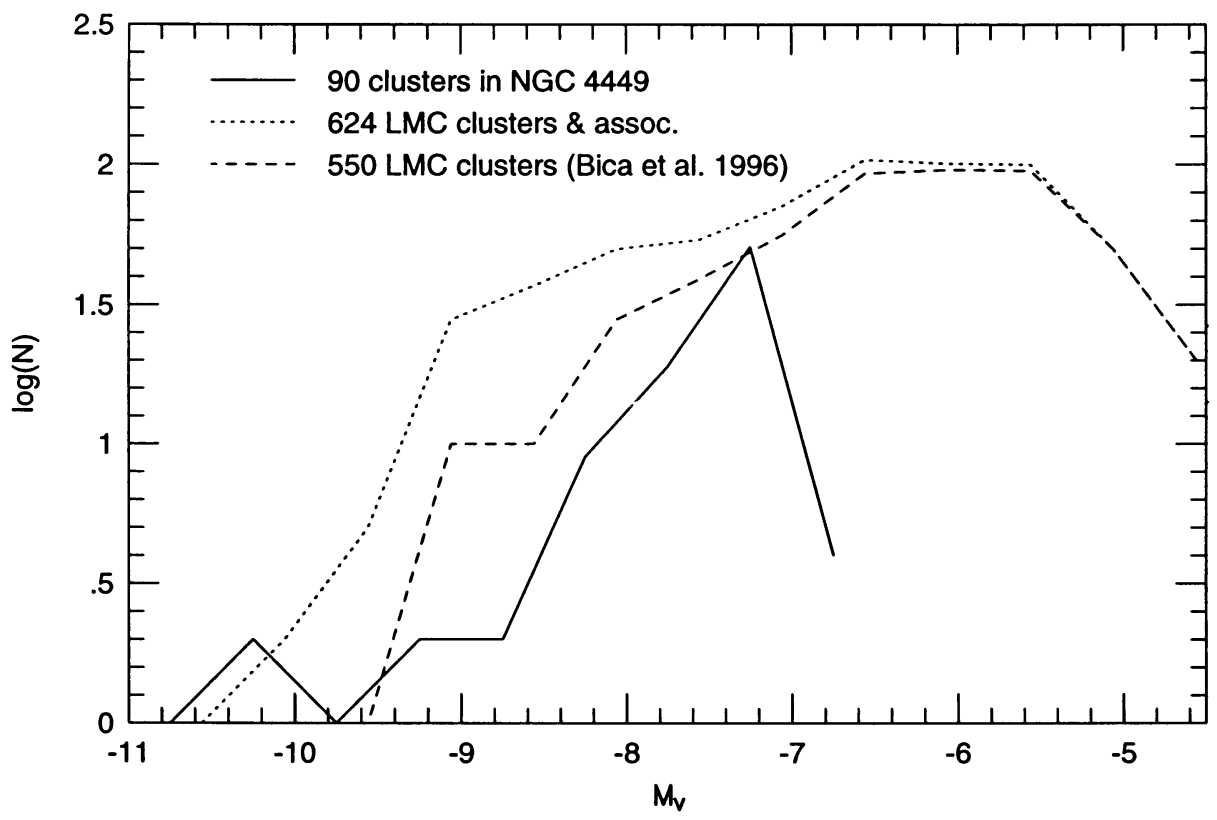

Figure 1. V band luminosity functions for clusters in NGC 4449 and the LMC.

The luminosity function is a reflection of the underlying mass function of the clusters, their age distribution, and metallicities. For the moment (lacking colors) we can say nothing about the ages and metallicities. But it is safe to conclude that NGC 4449 has a substantial population of bright clusters like the LMC. To within a scaling factor the cluster luminosity function is much like the LMC. On the basis of the recent starburst activity, we predict that the majority of these clusters will be found to have ages less than 1 Gyr.

\section{References}

Bothun, G.D. 1986, AJ, 91, 507

Harris, W.E. 1991, ARA\&A, 29, 543

Hill, R.S., Home, A.T., Smith, A.M., Bruhweiler, F.C., Cheng, K.P., Hintzen, P.M.N., \& Oliversen, R.J. 1994, ApJ, 430, 568

Hunter, D.A., \& Gallagher, J.S. 1997, ApJ, 475, 65

Hunter, D.A., Wilcots, E.M., van Woerden, H., Gallagher, J.S., \& Kohle, S. 1998, ApJ, 495, 47

Melisse, J.P., \& Israel, F.P. 1994, A\&AS, 103, 391 